\title{
SARS-CoV2 Related Multi System Inflammatory Syndrome in a Child with Chronic Kidney Disease: Case Report
}

\author{
Francesca Stefanachi ${ }^{1}$. Elisa Benetti ${ }^{1}$. Germana Longo ${ }^{1}$ - Mattia Parolin ${ }^{1} \cdot$ Claudia Maria Bonardi $^{2}$. \\ Davide Meneghesso ${ }^{1}$ (D)
}

Accepted: 16 June 2021 / Published online: 24 June 2021

(C) The Author(s), under exclusive licence to Springer Nature Switzerland AG 2021

\begin{abstract}
Since April 2020, several paediatric cases were reported with a multisystemic inflammatory syndrome related with SARS-CoV2, called MIS-C. In this case report, we describe a 2-year-old male with end-stage renal disease (ESRD) in renal replacement therapy (RRT) with peritoneal dialysis and severe hypertension affected by a severe SARS-CoV2 related illness characterised by multiorgan failure and need for intensive care, with clinical and instrumental features compatible with MIS-C. Most paediatric patients with kidney disease experience mild SARS-CoV2 disease and to our knowledge, this is the first case of a child with chronic kidney disease suffering from MIS-C. We believe that chronic kidney disease together with dialysis status and severe hypertension play a crucial role on developing severe forms of SARS-CoV2 related disease.
\end{abstract}

Keywords MIS-C $\cdot$ COVID $\cdot$ Coronavirus $\cdot$ Chronic kidney disease $\cdot$ Case report $\cdot$ Paediatric

\section{Introduction}

Since December 2019, SARS-CoV2 infection has rapidly spread worldwide causing a severe acute respiratory syndrome. Children and adolescents account for a small proportion of COVID-19 cases. Currently, among children, more than 2 million cases have been reported, according to American Academy of Pediatrics data [1]. Clinical manifestations in children are generally milder than in adults, with a lower hospitalisation rate and need for intensive care support [2]. Moreover, while chronic kidney disease (CKD) and hypertension (HT) have been described as important risk factors for severe SARS-CoV2 in adults [3], in the paediatric population, these comorbidities are apparently unrelated with a severe course of the disease $[4,5]$.

Since the outbreak of the COVID-19 pandemic, a severe multisystem inflammatory syndrome associated with SARSCoV2 infection, named MIS-C, characterised by fever,

Davide Meneghesso

davide.meneghesso@aopd.veneto.it

1 Department of Women's and Children's Health, Paediatric Nephrology, Dialysis and Transplant Unit, University Hospital of Padova, Padua, Italy

2 Department of Women's and Children's Health, Pediatric Intensive Care Unit, University Hospital of Padova, Padua, Italy systemic inflammation, and multiple organ failure, has increasingly been described in the paediatric population $[6,7]$.

In this article, we describe the first paediatric case of severe COVID-19, compatible with MIS-C, in a 2-year-old child with end-stage renal disease (ESRD) in renal replacement therapy (RRT) with peritoneal dialysis and severe arterial hypertension (HT).

\section{Case Presentation}

During COVID pandemic, a 2-year-old child affected by steroid-resistant nephrotic syndrome (genetic diagnosis still in progress), ESRD in peritoneal dialysis for 1 year and autoimmune hypocortisolism in glucocorticoid replacement therapy, was admitted to our Paediatric Unit for rapidly worsening severe HT in the past 2 months.

Recent past medical history was characterised by a fastsolving episode of severe acute respiratory syndrome and heart failure without any proved infective underlying cause; the episode occurred one month before admission and solved within $48 \mathrm{~h}$. The subsequent echocardiography showed: left ventricle hypertrophy with a mild septal dyskinesia, left ejection function (EF) of 52\% and persistence of dilation of left coronary vessels stable since previous control, all features compatible with a hypertensive heart disease. 
The patient underwent surgical nephrectomy because of persistent severe HT, resistant to multiple antihypertensive oral drugs, including ACE inhibitors, angiotensin receptor blockers, alpha-agonist, beta-blockers and calcium channel blocker (CCBs), and fenoldopam parenteral therapy. After surgery, antibiotic therapy was started and peritoneal dialysis continued regularly. Blood pressure control progressively improved. The antihypertensive therapy i.v. was discontinued 10 days after surgery; blood pressure was controlled with triple oral therapy with alpha-agonist, beta-blockers and CCBs.

During surgery-day, the mother resulted positive for SARS-CoV2 at molecular test. The day after surgery, the patient himself tested positive at nasopharyngeal swab, even if asymptomatic.

Three days after surgery and 2 days after his first positive swab, the patient experienced fever, rhinitis, sore throat, vomiting, diarrhoea and abdominal pain.

Five days after the first positive swab, fever became not responsive to antipyretic therapy and C-reactive protein markedly increased. Abdominal ultrasound was negative for postsurgical complications. Hydrocortisone replacement therapy was increased. On day six, the patient experienced dyspnoea and hypoxia with lung crackles and worsening hepatomegaly. Chest x-ray showed findings of interstitial pneumonia ("extended and shaded thickening mainly parahilar"). Initially ventilation support with high flow nasal cannula (HFNC) was started. However, one day later, a rapid clinical worsening occurred with severe dyspnoea and oxygen desaturation not responsive to oxygen therapy and maximisation of ventilation support with HFNC, associated with hypotension and tachycardia. Bedside echocardiography revealed left ventricle ejection function (LVEF) of 11-15\%, moderate mitral regurgitation and dilation of left heart with right ventricle pressed, with no cardiac or pleural effusion.

Patient required admission to the paediatric intensive care unit (PICU), invasive ventilation support and inotropic support therapy with milrinone. Antihypertensive therapy was stopped.

Lab tests demonstrated: anaemia ( $\mathrm{Hb} 8.1 \mathrm{~g} / \mathrm{L})$, lymphocytopenia (500 cells $/ \mathrm{mmc}$ ), mild thrombocytopenia $(82,000$ cells $/ \mathrm{mmc})$, elevated acute inflammation reactants (PCR $110 \mathrm{mg} / \mathrm{dL}$, ferritin $1611 \mu \mathrm{g} / \mathrm{L}$, D-dimer $714 \mu \mathrm{g} / \mathrm{L}$, IL2-R $7294 \mathrm{KU} / \mathrm{L}$, IL6 $93 \mathrm{ng} / \mathrm{L}$ ), increase of heart failure lab-index NT-proBNP $>175,000 \mathrm{ng} / \mathrm{L}$ with mild increase of troponin $4.7 \mathrm{ng} / \mathrm{L}$; abnormal liver function tests (AST $242 \mathrm{UI} /$ L, ALT $176 \mathrm{UI} / \mathrm{L})$ and increased LDH (665 U/L). The peripheral blood smear was negative for schistocytes. Abdominal ultrasonography revealed liver non homogeneous echopattern and periportal oedema.

On suspicion of SARS-CoV2 related disease, since the first day of PICU admission, we performed: steroid therapy (methylprednisolone $2 \mathrm{mg} / \mathrm{kg}$ /day i.v. once daily for five doses), azithromycin for three days, heparin i.v. (10 UI/ $/ \mathrm{kg} / \mathrm{h}$ c.i.) and hyperimmune plasma (100 mg/kg/die - three doses). The patient also required blood and albumin transfusions. Peritoneal dialysis was maximalised from $10 \mathrm{~h} /$ day to continuous dialytic treatment. Dextrose concentration of dialytic fluid and dwell time were modulated according to patient need. Cytokine filter hemodalysis was evaluated but not performed due to great need for filter priming with hemodynamically unstable patient.

After $24 \mathrm{~h}$ of mechanical ventilation, the echocardiography revealed fast improvement of heart function with LVEF increased to $45 \%$. Five days after intubation, milrinone was discontinued and antihypertensive therapy was restarted. The patient remained intubated for three more days, then was supported with non invasive ventilation for $24 \mathrm{~h}$ and finally with HFNC up to 7 days after extubation.

Finally, the patient experienced a progressive clinical improvement, HT was controlled by oral therapy, respiratory failure resolved and hepatomegaly reduced. After 18 days since the first positive swab and after 9 days since intubation, nasopharyngeal swab resulted negative for SARS-CoV2. Two weeks after intubation NT-proBNP decreased and echocardiography revealed a recovery of previous heart function.

\section{Conclusions}

Reports from Europe and North America described cases of children presenting a Multisystem Inflammatory Condition leading to multiorgan failure temporally related to SARS$\mathrm{CoV} 2$ infection.

Our patient experienced clinical manifestations and laboratory findings described in MIS-C developing an acute multiorgan failure (MOF). Lab tests revealed elevated labmarkers of inflammation, including C-reactive protein (CRP), D-dimer, ferritin, interleukin 6 (IL-6) and lymphocytopenia. MOF promptly resolved using supportive and immunosuppressive therapy.

From the SARS-CoV2 worldwide spread, only few evidence of SARS-CoV2 infection in children with chronic kidney disease were reported. A global study reported no case of severe illness or MIS-C in 113 children with kidney disease in immunosuppressive therapy [5]. Also, a Spanish retrospective study of 26 patients [8] and two case reports [9] described a mild clinical course of infection in children with kidney disease, with no case of PICU admission.

MIS-C is a novel condition and pathogenesis is not still well defined. Increasing evidence suggests a link between MIS-C and immune dysregulation. SARS-CoV2 infection, indeed, may lead to a deregulation of the immune response with increase of neutrophil blood level and decrease of Thelper and T-regulator and an overaction of proinflammatory cytokines, causing a multisystem inflammation and the subsequent multiorgan failure $[6,10]$. 
According to ECDC, it is still unknown why only a relatively small number of children have been reported with MIS$\mathrm{C}$ compared with the number of children with confirmed or suspected SARS-CoV2 infection [11].

To our knowledge this is the first clinical case of a child with CKD suffering from MIS-C. We hypothesise that patients with CKD, particularly if in presence of other comorbidities (i.e. severe HT, peritoneal dialysis), could have a proinflammatory systemic activation that may increase the risk of developing the inflammatory SARS-CoV2 related syndrome, revealing the immune pathogenesis of MIS-C.

Moreover, given that cardiovascular issues are emerging as frequent complications of SARS-CoV2 infection in children, patient with congenital heart diseases seem to be at risk for a severe form of COVID-19, because of their low basal cardiopulmonary reserve [11].

As described in this case report, paediatric patients with ESRD often experienced severe hypertension even resulting in hypertensive heart disease. So, in presence of severe hypertension, like children with congenital heart disease, paediatric patients with kidney disease could have an increased risk for severe SARS-CoV2 related illness provided by the preexisting cardiovascular impairment.

More studies are necessary to evaluate the role of ESRD and PAH as a risk factors for Multisystem Inflammatory Syndrome in children with COVID-19 (MIS-C).

Author Contribution FS and DM performed the literature search and wrote the first draft of the manuscript. All authors contributed to the critical appraisal and approved the final version of the manuscript that was submitted.

Data Availability Data will be made available upon request.

Code Availability Not applicable.

\section{Declarations}

Ethics Approval and Consent to Participate Not applicable.

Consent for Publication Not applicable.

Conflict of Interest The authors declare no competing interests.

\section{References}

1. American Academy of Pediatrics - State-level data report. (accessed Jan 3rd, 2021)
2. Ludvigsson JF. Systematic review of COVID-19 in children shows milder cases and a better prognosis than adults. Acta Paediatr. 2020;109(6):1088-95. https://doi.org/10.1111/apa.15270 Epub 2020 Apr 14.

3. ERA-EDTA Council; ERACODA Working Group. Chronic kidney disease is a key risk factor for severe COVID-19: a call to action by the ERA-EDTA. Nephrol Dial Transplant. 2021;36(1):87-94. https://doi.org/10.1093/ndt/gfaa314.

4. Mastrangelo A, Morello W, Vidal E, Guzzo I, Annicchiarico Petruzzelli L, Benetti E, et al. COVID-19 Task Force of the Italian Society of Pediatric Nephrology; COVID-19 Task Force of the Italian Society of Pediatric Nephrology. Impact of COVID19 pandemic in children with CKD or immunosuppression. Clin J Am Soc Nephrol. 2020:CJN.13120820. https://doi.org/10.2215/ CJN.13120820 Epub ahead of print.

5. Marlais M, Wlodkowski T, Al-Akash S, Ananin P, Bandi VK, Baudouin V, et al. COVID-19 in children treated with immunosuppressive medication for kidney diseases. Arch Dis Child. 2020. https://doi.org/10.1136/archdischild-2020-320616 Dec 21: archdischild-2020-320616. Epub ahead of print.

6. Jiang L, Tang K, Levin M, Irfan O, Morris SK, Wilson K, et al. COVID-19 and multisystem inflammatory syndrome in children and adolescents. Lancet Infect Dis. 2020;20(11):e276-88. https:// doi.org/10.1016/S1473-3099(20)30651-4 Epub 2020 Aug 17.

7. Henderson LA, Canna SW, Friedman KG, Gorelik M, Lapidus SK, Bassiri H, et al. American College of Rheumatology clinical guidance for pediatric patients with multisystem inflammatory syndrome in children (MIS-C) associated with SARS-CoV-2 and hyperinflammation in COVID-19. Version 2. Arthritis Rheum. 2020 Dec 5. https://doi.org/10.1002/art.41616 Epub ahead of print.

8. Melgosa M, Madrid A, Alvárez O, Lumbreras J, Nieto F, Parada E, et al. Spanish Pediatric Nephrology Association. SARS-CoV-2 infection in Spanish children with chronic kidney pathologies. Pediatr Nephrol. 2020;35(8):1521-4. https://doi.org/10.1007/s00467-02004597-1 Epub 2020 May 20.

9. Rawson A, Wilson AC, Schwaderer AL, Spiwak E, Johnston B, Anderson S, et al. Coronavirus disease 2019 (COVID-19) in two pediatric patients with kidney disease on chronic immunosuppression: a case series. Hemodial Int. 2020;5. https://doi.org/10.1111/ hdi. 12876.

10. Rodriguez-Gonzalez M, Castellano-Martinez A, Cascales-Poyatos HM, Perez-Reviriego AA. Cardiovascular impact of COVID-19 with a focus on children: a systematic review. World J Clin Cases. 2020;8(21):5250-83. https://doi.org/10.12998/wjcc.v8.i21. 5250 .

11. European Centre for Disease Prevention and Control. Rapid risk assessment: paediatric inflammatory multisystem syndrome and SARS-CoV-2 infection in children. 2020. https://www.ecdc. europa.eu/ en/publications-data/paediatric-inflammatorymultisystem-syndrome-and-sars-cov-2-rapid-risk-assessment (accessed July 16, 2020).

Publisher's Note Springer Nature remains neutral with regard to jurisdictional claims in published maps and institutional affiliations. 